\title{
The Chant Meteor Procession of 1913 - Towards a Descriptive Model
}

\author{
Martin Beech ${ }^{1,2}$, Mark Comte ${ }^{2}$ \\ ${ }^{1}$ Campion College at the University of Regina, Saskatchewan, Canada \\ ${ }^{2}$ Department of Physics, The University of Regina, Saskatchewan, Canada
}

Email address:

beechm $@$ uregina.ca (M. Beech), comte20m@uregina.ca (M. Comte)

To cite this article:

Martin Beech, Mark Comte. The Chant Meteor Procession of 1913 - Towards a Descriptive Model. American Journal of Astronomy and Astrophysics. Vol. 6, No. 2, 2018, pp. 31-38. doi: 10.11648/j.ajaa.20180602.11

Received: April 5, 2018; Accepted: April 19, 2018; Published: June 28, 2018

\begin{abstract}
From an observational standpoint the Chant Meteor Procession of 9 February, 1913 is particularly remarkable, being especially noted for its long ground track of at least $15,000 \mathrm{~km}$, and for the slow motion and near parallel to the horizon paths adopted by the meteors. The circumstances surrounding the Procession are re-considered here in terms of the successive entry of multiple meteoroid clusters. These clusters are in turn considered to be derived from a temporarily captured Earth orbiting object that has undergone disaggregation. It is suggested that the general observational accounts of the Procession can be explained through the sequential entry of multiple meteoroid clusters that moved through the Earth's atmosphere on grazing-incident trajectories. It is further suggested that the parent object to the Procession, prior to its breakup, may have been no more than 3 to $4-\mathrm{m}$ across.
\end{abstract}

Keywords: Natural Earth Satellites, Meteoroid Ablation, Grazing Atmospheric Flight

\section{Introduction}

The Chant Meteor Procession occurred in the early evening hours of 9 February, 1913. The event was witnessed by many hundreds, if not thousands, of eye-witnesses from both sides of the Canada-US border and as a meteoritic phenomenon it has long been the topic of debate and speculation. The fireball observations were first investigated in detail by Clarence Chant $[1,2]$ and the exceptional nature of the Procession was soon realized. The first sighting of numerous fireballs was reported from the towns of Pense and Mortlach in southwestern Saskatchewan, Canada and additional reports were received from as far east as the island of Bermuda and by several ships located off the northeastern coast of Brazil [3-7]. The existing observations indicate a remarkably long ground track of at least $15,000 \mathrm{~km}$ in length for the Procession, and in turn this provides a distinct challenge for the construction of a viable physical explanation. Key to unraveling the Procession, it is argued here, is to view it as a distinct chain of related events. That is, it was not the result of one large and successively fragmenting meteoroid, or even many individual meteoroids of varying mass that entered the atmosphere simultaneously, nor was it a meteor shower. Rather, it was an extended sequence of meteoroid clusters that entered the Earth's atmosphere, at a shallow angle of trajectory, at successively different times during the course of the display. The parent body of the various meteoroid groups therefore, it is argued, broke apart many weeks to months before the Procession actually occurred, with the various components and meteoroid clusters being spread out along an arc at least $15,000 \mathrm{~km}$ in length.

\section{The Observations}

Eye-witness accounts collected by Chant, Denning and Mebane [1-7] not only indicate that the Procession was apparently continuous, as expressed by reports from Saskatchewan to at least Bermuda, but that it had the same general appearance from one location to the next. Essentially all observers describe the display as consisting of numerous meteors travelling parallel not only to each other but also to the horizon, and while eye-witness accounts provide less than ideal data to work with the only apparent changes reported from one location to the next was the total number of meteors 
seen. Figure 1 shows a composite image constructed from the data and eye-witness sketches obtained by Chant [1]. The approximate ground track is shown in red, and sketches from observers located in Ontario, Canada (at Parry Sound, Toronto, Kitchener, and Centreton) and from Bermuda (Hamilton) are superimposed. Also shown on the map are star-symbols which indicate locations from which sound (either sonic and/or electrophonic) were reported. It is argued here that the telling feature of the various accounts is not so much the number of individual meteors and/or their brightness, but the fact they moved in apparent groupings, and that all of the various meteors moved on a path essentially horizontal to the horizon. Eye-witness accounts of the duration times of fireballs and the arc lengths traveled on the sky are notoriously poor and difficult to work with, but Chant [1, 2], Denning [3] and Pickering [4, 5] all determine a characteristic velocity of about $8 \mathrm{~km} / \mathrm{s}$ and a near constant atmospheric path height of $50 \pm 10 \mathrm{~km}$ altitude. Under these circumstances an observer seeing the Procession moving from horizon to horizon, and passing through their zenith at the mid point, would witness a procession lasting some 3 minutes in duration. The account of J. E. Skidmore from Cobourg, Ontario seems particularly apropos: "they glided along so leisurely and did not seem to be falling as meteors usually do, but kept a straight course about $45^{\circ}$, or a little more, above the horizon. Our first impression was that a fleet of illuminated air-ships of monstrous size were passing. The incandescent fragments themselves formed what to us looked like the illuminations, while the tails seemed to make the frame of the machine. Sometimes there would be just a single collection, forming a single ship; then in a half-minute several collections would pass, looking like ships traveling in company. It took fully 3 minutes to pass. There was no noise; only beauty, beauty!" [1].

The velocity determination of about $8 \mathrm{~km} / \mathrm{s}$ for the meteors seems particularly telling with respect to the encounter conditions, since it suggests that the meteoroids in the procession were moving along temporary Earth-orbit captured trajectories. The orbital velocity $V_{\text {orb }}$ at height $h$ above the Earth's surface is

$$
V_{\text {orb }}=\left(1-\frac{1}{2} \frac{h}{R}\right) \sqrt{\left(\frac{G M}{R}\right)}
$$

Where $M$ and $R$ are the mass and radius of the Earth. For $h$ $=50 \mathrm{~km}$, so $V_{\text {orb }}=7.9 \mathrm{~km} / \mathrm{s}$. Such temporary capture events, taking place while the meteoroid moves through the Earth's atmosphere, undergoing active ablation, are not common, but they have been observed. The conditions that favor the occurrence of such events are a low angle of entry to the horizon, low initial entry speed, and a small physical size [8].

Sounds were reported from many locations along the Procession track (see figure 1). An observer in Warren, northwestern Minnesota recorded that upon seeing a brilliant body light-up the sky a rumble sound like that of railway trains or distant waterfalls was heard. Observers in southern Ontario and northern Pennsylvania, as well as in New York state, reported hearing rumbling sounds like cannon-fire, or like that of thunder, up to several minutes after the meteors were seen. Other observers reported hearing 'swishing' sounds or a 'hiss' like that of a fire-rocket at the same time that the Procession was visible. Observers onboard the sailing ship Ponape, located off Rio Grande de Norte, Brazil, also reported hearing sounds [7]. Such reports are entirely consistent with other fireball events and they may be attributed to sonic booms and the production of electrophonic sounds [9-13]. The production of sonic booms is particularly interesting since it indicates that meteoritic material more than likely did reach the ground, although no meteorites were actually recovered.

\section{A Preliminary Model}

Given the more than 100 years that have passed since the Procession took place, and the complete lack of any instrumental data, it is not possible to directly model the event in detail. This being said, a model that accounts for the greater bulk of the observations, such as they are, can be constructed and the circumstances leading-up to the appearance of the display may at least be assessed. The idea that the Procession was something all together different from the passage of a single, large, fragmenting meteoroid passing through the atmosphere was voiced early-on [2, 3, 4]. Indeed, both Chant [1] and Denning [3] argued that the objects forming the Procession were captured Earth satellites. In 1939, C. C. Wylie [14] suggested that the event was a "normal meteor shower" - a suggestion that O'Keefe [15] strongly argued against in 1959. Indeed, O'Keefe suggested that the fireballs were derived from a circum-terrestrial ring of material formed through the ejection of material from lunar volcanoes (he additionally suggested that the Procession should be named the Cyrillid meteor shower, since the event took place on the feast day of Saint Cyril of Alexandria). In 1964 O'Keefe [16] additionally suggested that a link might exist between the Cyrillid's and terrestrial tektites, the latter being derived from asteroid impacts upon the Moon. O'Keefe's ideas on both tektite origins and the Cyrillid shower have not proved popular, and they are generally dismissed in terms of revised ideas in the modern era. For all this, however, the idea that the 1913 Procession is related to the Earth undergoing an encounter with a string of natural satellites is still robust [17]. Indeed, Granvik, Vaubaillon and Jedicke [18] have argued that the Chant Meteor Procession has its origins within a sub-group of nearEarth objects (NEOs) that can produce temporarily captured orbiters (TCOs) or minimoons. Granvick and co-workers estimate that there is at least one 1-m sized TCO in the nearEarth environment at any one time. The capture rate for 5 $10 \mathrm{~m}$ sized TCOs is estimated to be on a decadal scale, while $100 \mathrm{~m}$ sized TCOs are only likely to be encountered on timescales of order 100,000 years. Furthermore, Granvick and colleagues find that perhaps of order $0.1 \%$ of all meteoroids that enter the Earth's atmosphere are TCOs. In addition, Granvick et al., argue that the most likely time for TCO capture is when Earth is near perihelion (from late 
December, through January to early February) and that the typical Earth-atmosphere encounter speed of TCOs will be of order the Earth's escape velocity $\sim 11.3 \mathrm{~km} / \mathrm{s}$.

In principle every planet within the solar system has the potential for acquiring TCOs. Fedorets, Granvik and Jedicke [19], for example, argue that comet Shoemaker-Levey-9 (comet D/1993 F2) was a TCO of Jupiter for some 25 years before the various components of its fragmented nucleus plunged into the planet's upper cloud deck in July of 1994. The only confirmed terrestrial TCO is that of asteroid 2006 $\mathrm{RH}_{120}$; a NEO with a heliocentric orbit very similar to that of Earth [20]. This 2-3-meter sized object undergoes a close encounter with the Earth-Moon system approximately every 20 years and it was in fact discovered as a TCO - a status that it held from September 2006 to June 2007. After leaving Earth orbit in 2017, $2006 \mathrm{RH}_{120}$ entered into an Amor-class NEO orbit. Its next close encounter with the Earth will be in 2028. A short, one-month duration TCO designation has additionally been applied to the NEO $1991 \mathrm{VG}$, and this 5 to 10-meter sized object is the potential target of NASA's Near Earth Asteroid Scout mission (a CubeSat and solar sail spacecraft system) presently scheduled for launch in late 2019. The bright European Network fireball EN130114, observed over Europe on 13 January, 2014 has additional been labeled as a TCO prior to Earth impact [21] - this object entered the Earth's atmosphere at a relatively low angle of 33 degrees to the horizon, had an estimated mass of $5 \mathrm{~kg}$, giving it a diameter of some $15-\mathrm{cm}$, and an atmospheric entry speed that was just $11.2 \mathrm{~km} / \mathrm{s}$.

The Chant Meteor Procession of 13 February 1913 appears to be a good candidate object for TCO status. The time of the event, early February, and its slow apparent speed are consistent with the predictions outlined by Granvick et al. [18]. Here it is suggested, however, that the parent object broke apart before the individual fragments, or meteoroid clusters, encountered the Earth's atmosphere along shallowangle trajectories. The parent object is taken therefore to have had a loose, rubble pile, structure and it is supposed that at some stage as it orbited the Earth it passed close to or even within the Earth's Roche radius - that is within 200,000 km of the Earth - and gently separated-out into a string of fragments and meteoroid clusters. It was the entry of these fragments, in a staggered and sequential fashion, that resulted in the appearance of the Chant Procession. The details of this scenario are investigated below. Firstly, the atmospheric flight and meteoroid ablation conditions are considered. Second, a series of possible model outcomes are described, and thirdly a discussion for future work is presented.

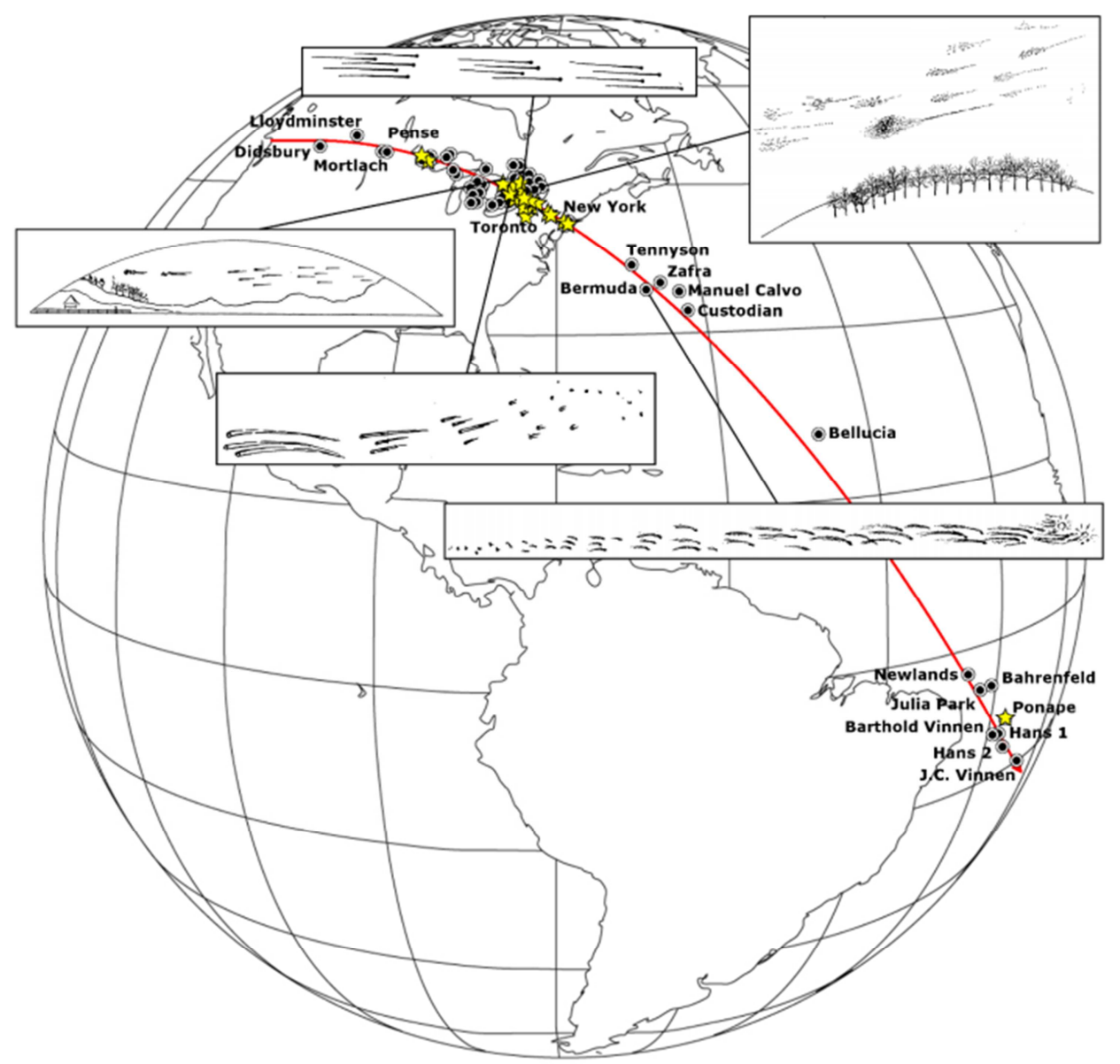

Figure 1. Schematic of the ground track for the Chant Meteor Procession (red line). The locations from which various eye-witness reports were collected [1$7]$ are shown by small black dots. Locations from which either sonic booms or electrophonic sounds were reported are indicated by yellow stars. The inset drawings of the Procession are taken from various eye-witness accounts [from ref. 1], and they illustrate the common description that the meteors moved in clusters and along trajectories that ran parallel to the horizon. 


\section{The Equations of Meteoroid Ablation}

Within the context of a non-plane-parallel atmosphere, the equations of meteoroid ablation and motion are as follows [22]:

$$
\begin{gathered}
\frac{d V}{d t}=-\Gamma \rho_{\text {atm }}\left(\frac{A}{m}\right) V^{2}+g \sin (\theta) \\
\frac{d m}{d t}=-\left(\frac{\Lambda}{2 \zeta}\right) \rho_{a t m} A V^{3}\left(\frac{V^{2}-V_{\text {dark }}^{2}}{V^{2}}\right) \\
\frac{d \theta}{d t}=\left(\frac{1}{m V}\right)\left(m g \cos (\theta)-\frac{1}{2} C_{\text {lift }} \rho_{\text {atm }} A V^{2}\right)-\frac{V \cos (\theta)}{R+h} \\
\frac{d h}{d t}=-V \sin (\theta) \\
\frac{d X}{d t}=\frac{V \cos (\theta)}{1+h / R}
\end{gathered}
$$

where $m$ is the meteoroid mass, $V$ is the meteoroid velocity, $A$ is the surface area of the meteoroid presented to the oncoming airflow, $g$ is the acceleration due to gravity at height $h, R=6371 \mathrm{~km}$ is the radius of the Earth, $\rho_{a t m}$ is the atmospheric density at altitude $h, \theta$ is the flight angle to the horizon, $C_{\text {lift }}$ is the lift coefficient (taken as being zero in this study), and $\zeta$ is the enthalpy of melting and vaporization. The term $V_{\text {dark }}$ in equation (3) is the dark-flight velocity limit, such that for $V<V_{\text {dark }}=2 \mathrm{kms}^{-1}$ vigorous mass loss via ablation is assumed to have stopped. Equation (6) describes the down-range, ground track distance $X$ along the Earth's surface. Rather than being assumed constant, the drag coefficient $\Gamma$ and the heat transfer coefficient $\Lambda$ are evaluated according to the characteristics of the oncoming airflow and the atmospheric height. Using the Reynolds number $R e$ as the regime defining parameter, the drag coefficient is evaluated as:

$$
\begin{gathered}
\Gamma=\frac{24}{\operatorname{Re}}\left[1+\frac{3}{16} \operatorname{Re}\right], \text { when } \operatorname{Re}<1 \\
\Gamma=\frac{24}{\operatorname{Re}}\left[1+0.15 \operatorname{Re}^{0.687}\right], \text { when } 1<\operatorname{Re}<10^{3} \\
\Gamma=0.4 \text {, when } \operatorname{Re}>10^{3}
\end{gathered}
$$

where $R e=L V / \mathrm{v}$, with $L$ being the characteristic dimension of the meteoroid (taken to be its diameter), $V$ is the velocity of the meteoroid through the atmosphere, and $v$ is the kinetic viscosity of the atmospheric gas. Equation (7) is the Ossen approximation to the classic Stokes law formula, while (8) is taken from Clift et al. [23]. Equation (9) is the limiting value for the high Reynolds number value to the drag coefficient for a sphere. The heat transfer coefficient is taken from the formulation of Melosh and Goldin [24] with:

$$
\Lambda=\frac{8}{\gamma}\left(\frac{\mathrm{Nu}}{\operatorname{Re} \operatorname{Pr}}\right) \frac{1}{\Gamma}
$$

where $\gamma=1.4$ is the ratio of specific heats for air, $N u$ is the Nusselt number, and $\mathrm{Pr}$ is the Prandtl number. Melosh and Goldin [24] provide formula for evaluating the Nusselt number (defined as the ratio of the convective to radiative heat transfer) in terms of the Mach number $M=V / c$, where $V$ is the velocity of the meteoroid and $c=\sqrt{\gamma R T_{a t}}$ is the atmospheric sound speed, with $R=287 \mathrm{~J} / \mathrm{K} / \mathrm{kg}$ being the gas constant for air, and $T_{a t}$ being the atmospheric temperature. The Prandtl number $\operatorname{Pr}=C_{P} \mu / k=0.72$ is the ratio of the kinetic viscosity to the thermal diffusivity. In evaluating the Prandtl number $\operatorname{Pr}$ the specific heat of air is taken to be $C_{P}=$ $1005 \mathrm{~J} / \mathrm{kg}$, the dynamic viscosity is taken to be $\mu=1.8 \times 10^{-5}$ $\mathrm{Ns} / \mathrm{m}^{2}$, and the thermal conductivity is set as $k=0.025$ $\mathrm{W} / \mathrm{mK}$. The atmospheric density variation with height is determined via a least squares polynomial fit to the NRLMISE-00 [25] atmosphere model sequenced at $5 \mathrm{~km}$ intervals over the range 0 to $400 \mathrm{~km}$ in altitude. The NRLMISE-00 model is also used to evaluate the atmospheric sound speed, via a series of least square polynomial equations in height constructed so as to describe the variation in atmospheric temperature $T_{a t}$.

A meteoroid will undergo fragmentation during its atmospheric flight if the ram pressure $P_{\text {ram }}$ of the on-coming airflow exceeds the compressive strength $\sigma_{\text {com }}$ of its constituent material. Accordingly, the numerical code follows the variation in the ram pressure expressed as: $P_{\text {ram }}=\Gamma \rho V^{2}$, where $V$ is the velocity, $\Gamma$ is the drag coefficient and $\rho$ is the atmospheric density. Once $P_{\text {ram }}>\sigma_{\text {com }}$ fragmentation is assumed to occur. Characteristic values for which meteoroids have been observed to fragment correspond to $\sigma_{\text {com }}$ being of order 1 to $10 \mathrm{MPa}$ [26]. Our simulations additionally track and quantify the heights between which electrophonic sound generation might proceed [13, 27]. This phenomenon is possible once the Reynolds number $R e>10^{6}$, that is the flow is turbulent, and when the magnetic Reynolds number Rem = $V \tau_{\mathrm{m}} / D>D / 10$, where $D$ is the meteoroid diameter and $\tau_{\mathrm{m}}$ is the characteristic decay time for the diffusion of the magnetic field [27]. This onset condition can be cast in terms of the height $h$ of the meteoroid being lower than the transition height $h_{\text {trans }}$, where, $h_{\text {trans }}=-H \ln \left(\operatorname{Re} \mu_{0} / V \rho_{0} D\right)$, where $H$ is the atmospheric scale height, $\rho_{0}$ is the atmospheric density at sea-level, and $\mu_{0}$ is the dynamic viscosity.

\section{Model Calculations}

In the following simulations it is assumed that the meteoroid entry velocity is $12 \mathrm{~km} / \mathrm{s}$, that is, just above Earth's escape velocity and consistent with the predicted TCO Earth encounter speed [18]. Furthermore, a constant density for the meteoroid material of $\rho_{\text {met }}=3400 \mathrm{~kg} / \mathrm{m}^{3}$ is adopted, this being characteristic of that expected for chondritic meteorites, and the starting height for each 
simulation is taken to be $300 \mathrm{~km}$ in altitude. The initial meteoroid mass and angle of entry into the atmosphere are taken as model variables to be chosen. The modeling approach adopted has been to determine the range of the parameter space in mass and entry angle that allows for long, near parallel to the horizon trajectories with a height of order $50 \pm 10 \mathrm{~km}$ altitude and a characteristic speed of $8 \pm 1 \mathrm{~km} / \mathrm{s}$. An object moving under such conditions would represent just one component in the Procession. Figure 2 shows the atmospheric height versus range variation for a series of calculations relating to a meteoroid having an initial mass of $1000 \mathrm{~kg}$ (diameter of $0.82 \mathrm{~m}$ ) entering the Earth's atmosphere at an angle of just over 12 degrees to the local horizon (at $300 \mathrm{~km}$ altitude). Figure 3 shows the velocity variation with range associated with the $1000 \mathrm{~kg}$ meteoroid. Meteoroids having an initial mass of $500 \mathrm{~kg}$ (diameters of $0.7 \mathrm{~m}$ ), with an entry velocity of $12 \mathrm{~km} / \mathrm{s}$ behave in a similar manner to that portrayed in figures 2 and 3, although, for the same range of initial entry angles, these meteoroids can attain ground paths in excess of $2500 \mathrm{~km}$ while situated in the altitude range $50 \pm$ $10 \mathrm{~km}$.

For the $1000 \mathrm{~kg}$ mass meteoroid simulations, irrespective of whether ground impact or atmospheric escape occurred, the ground path length within the height range of interest is $X$ $\approx 1600$ to $2000 \mathrm{~km}$. In these same simulations the calculated ram pressure at no time exceeded $1 \mathrm{MPa}$ suggesting that under the adopted conditions no fragmentation is likely. For the atmosphere escape tracks show in figure 2, the velocity of the meteoroids in the altitude range of interest is $\sim 8.5 \mathrm{~km} / \mathrm{s}$ (as revealed in figure 3), which is characteristic of that deduced from the eyewitness accounts. Given a typical ground path length of order $1600 \mathrm{~km}$, the entire ground path of the Procession, if always populated by such meteoroids, would require a total set of some 10 to $15,1000 \mathrm{~kg}$ meteoroids. Such a minimally populated Procession would require the pre-Earth encounter disruption of a body having an initial mass of about $15,000 \mathrm{~kg}$ with an initial diameter of about 2 meters. If the Procession is assumed to contain some 100 , say, $1000 \mathrm{~kg}$ meteoroids then the size of the parent object is increased to about 4 meters across. The arc length along which the various meteoroid clusters, from first to last, would need to be spread over prior to encountering the Earth's atmosphere will be of order the Procession ground path length of $15,000 \mathrm{~km}$. Assuming a gentle separation of components during the break-up of the parent asteroid, with the components having, say, a characteristic separation speed of a few meters per second, then our putative string of meteoroid clusters would take several months to fully form.

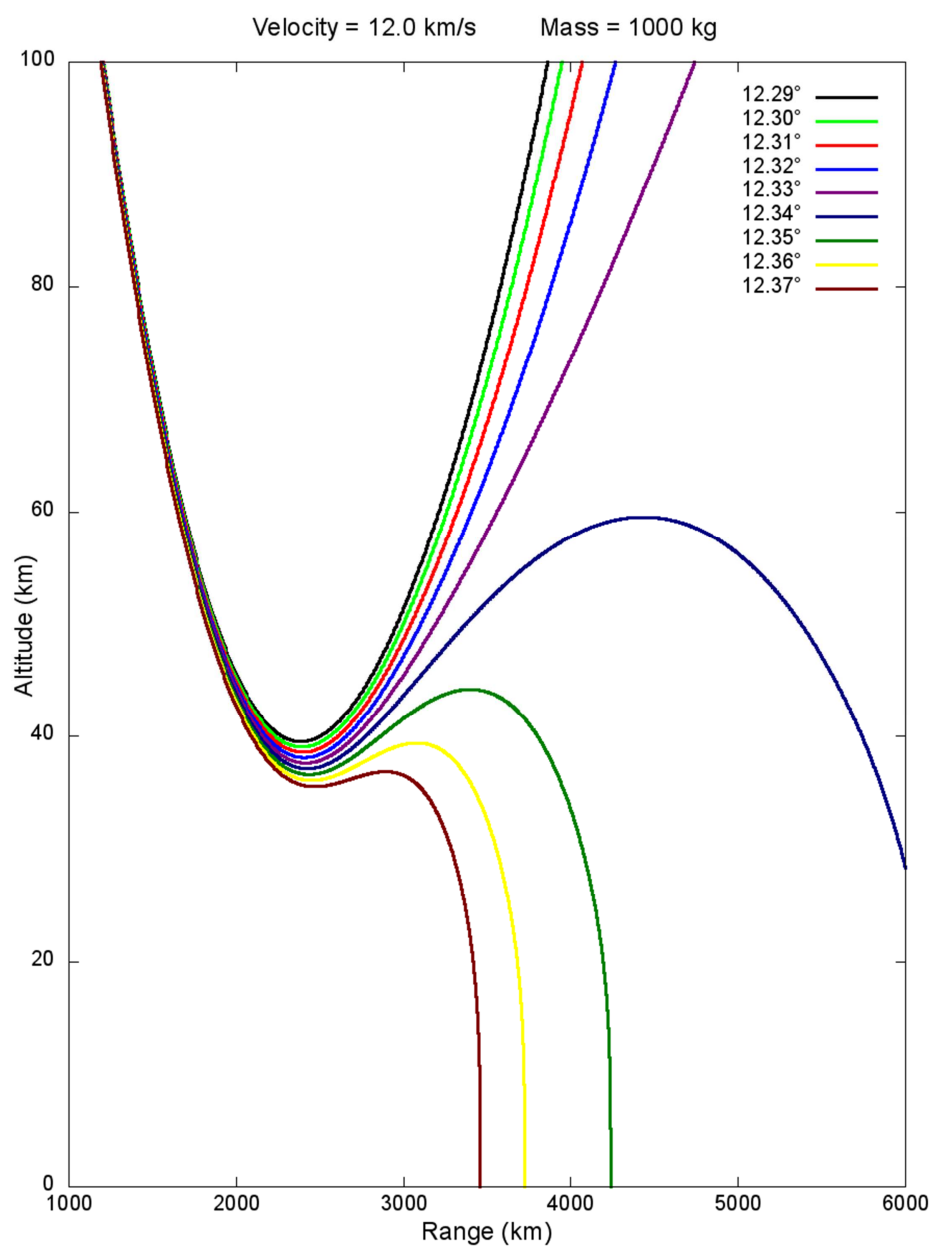

Figure 2. Atmospheric flight characteristics for a $1000 \mathrm{~kg}$ meteoroid entering Earth's atmosphere at $12 \mathrm{~km} / \mathrm{s}$. Each curve is labelled according to the initial angle of entry at $300 \mathrm{~km}$ altitude. For $\theta=12.33$ degrees, the meteoroid returns to cislunar space with a velocity of $8.6 \mathrm{~km} / \mathrm{s}$. For $\theta>12.34$ degrees the meteoroid penetrates deep into the atmosphere and potentially delivers meteoritic material to the ground. 


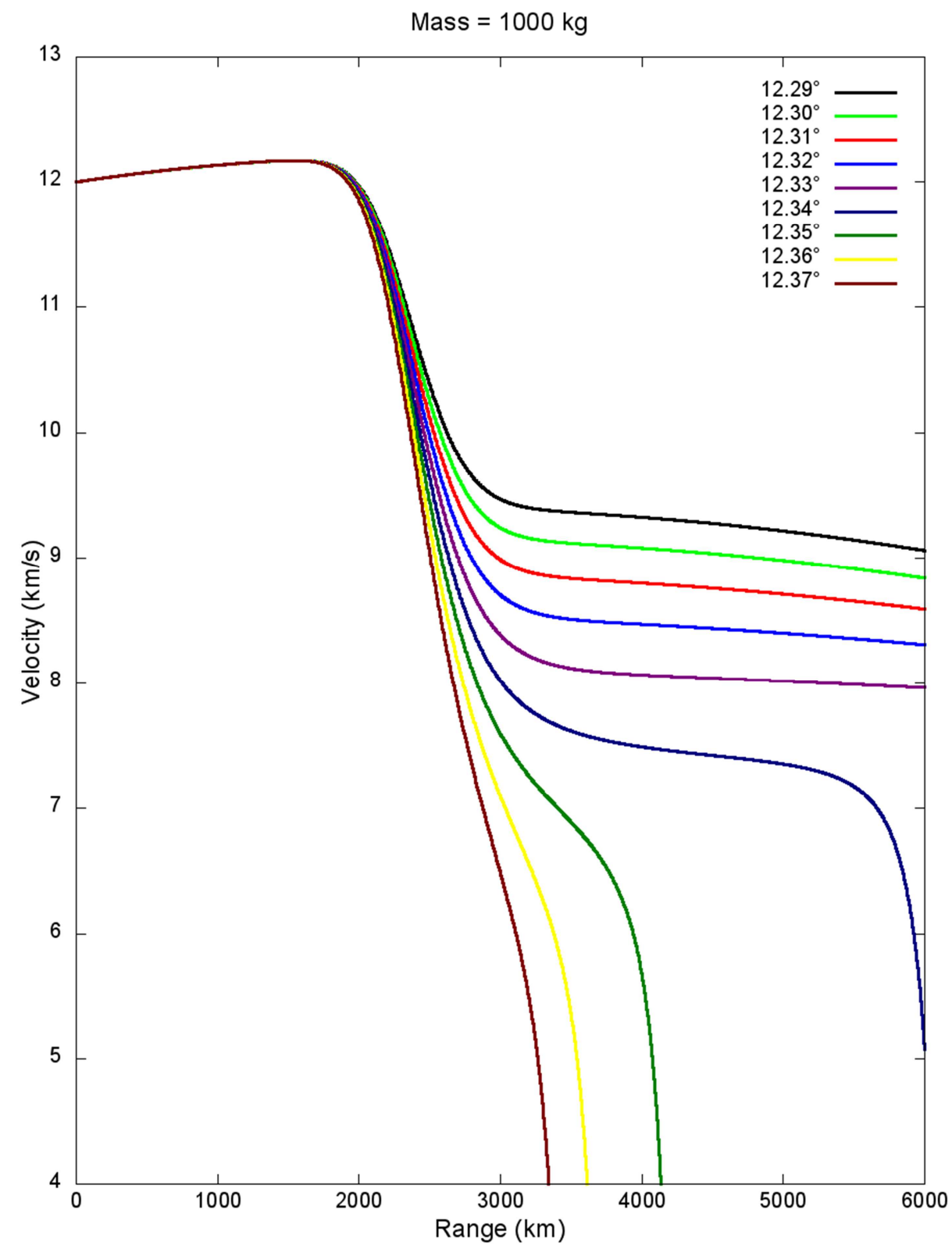

Figure 3. Velocity versus range variation for a $1000 \mathrm{~kg}$ meteoroid entering Earth's atmosphere at $12 \mathrm{~km} / \mathrm{s}$. Each curve is labelled according to the initial angle of entry at $300 \mathrm{~km}$ altitude.

The closest approach distance of a meteoroid to the Earth's surface $h_{\mathrm{ca}}$ (assuming no atmospheric interaction) is calculated at an altitude of $100 \mathrm{~km}$ and evaluated as: $h_{\mathrm{ca}}=$ $R\left[(1+100 / R) \cos \left(\theta_{100}\right)-1\right]$, where $R=6371 \mathrm{~km}$ is the Earth's radius and $\theta_{100}$ is the flight angle to the horizon at 100 $\mathrm{km}$ altitude. It is observed from the simulation calculations that for those meteoroids with initial masses in the range 500 $<m(\mathrm{~kg})<5000$, there is a small window, just a few kilometers wide, between $62<h_{\text {ca }}(\mathrm{km})<65$ that separates out whether a meteoroid will escape back into space or impact upon the ground. Meteoroids that pass through this window, however, will move along trajectories that result in meteors moving along near horizontal tracks at a near constant height. In agreement with Hills and Goda [8] it is found that this 'capture window' shrinks rapidly in size with increasing entry velocity, all other parameters being held constant. The ram pressure for even our largest mass simulation, with $m=5000 \mathrm{~kg}$, did not exceed $1 \mathrm{MPa}$ and accordingly no significant fragmentation would be expected during atmospheric flight. This result is consistent with eyewitness accounts [ $1-7]$, where, in general, it is reported that the meteors moved as single (non-fragmenting) entities. For just a small increase in the entry angle, say $\theta=15$ degrees, it is found that the maximum ram pressure for the $5000 \mathrm{~kg}$ meteoroid is of order $6.5 \mathrm{MPa}$, and accordingly fragmentation would be likely; the maximum ram pressure experienced by a $1000 \mathrm{~kg}$ meteoroid with this slightly steeper entry angle is found to be of order $4 \mathrm{MPa}$, again indicating a high probability of fragmentation. It is additionally found that once the entry angle allows for deep atmosphere penetration then the onset condition for electrophonic sound generation is readily satisfied. 
To sum-up, it has been found from a series of numerical simulations that objects encountering the Earth's upper atmosphere at $12 \mathrm{~km} / \mathrm{s}$, with a flight angle of about 6 degrees to the horizon at $100 \mathrm{~km}$ altitude, will move along extended, temporary Earth-orbit capture trajectories, within the height range $50 \pm 10 \mathrm{~km}$ altitude, that vary in length from about $2500 \mathrm{~km}$ (at $500 \mathrm{~kg}$ initial mass) to about $1400 \mathrm{~km}$ (at 5000 $\mathrm{kg}$ initial mass). Just a small increase in the entry angle will result in meteoritic material finding its way to the ground that such circumstances were actually realized is evidenced by the reports of sonic booms and electrophonic sounds, with such phenomenon requiring the deep penetration of a relatively large meteoroid into the lower Earth atmosphere. That no meteorites were recovered following the passage of the Procession is either a result of the relatively sparse human population (compared to the present day) along the ground track, or that only small (and hence not easily found) meteorites were produced - perhaps both of these possibilities held sway. In contrast to the meteorite producing situation, just a small decrease in the entry angle that allows for temporary capture will result in a meteoroid skipping-out of the atmosphere. Such fireballs present an interesting situation in that their atmosphere exiting velocity will be less than the Earth's escape velocity and accordingly they will re-enter the atmosphere at a later time. Atmosphere skipping fireballs are not commonly recorded, but they have been observed perhaps the best studied such fireball is that of 10 August, 1972 [28]. This latter object, with an estimated diameter of about 3-meters, entered Earth's atmosphere at a speed of 15 $\mathrm{km} / \mathrm{s}$ and descended to an altitude of $58 \mathrm{~km}$ before returning back into space.

\section{Conclusions}

The collected eye-witness accounts of the Chant Meteor Procession, while always emphasising the slow, extended and near horizontal flight of the individual meteor clusters, rarely emphasised the brightness, and this suggests that the meteoroid masses must have been relatively small, since the larger the meteoroid mass so the brighter will it appear for a given velocity. Accordingly, it seems appropriate to populate the initial chain of meteoroid groups by a series of perhaps, say, 50, $1000 \mathrm{~kg}$ mass meteoroids, and at least an equal number of $500 \mathrm{~kg}$ meteoroids, moving in unison but spread over an arc of some $15,000 \mathrm{~km}$ in length prior to encountering the Earth. The mass and diameter of a parent object capable of producing such a fragment chain would be of order $75,000 \mathrm{~kg}$ and 3.5 meters respectively. Obviously, a larger initial mass body could provide more fragments and possibly larger ones, than the numbers just presented. Unfortunately, however, there is no observational data to help us constrain the total number of fragments that contributed to the Chant Meteor Procession, and the numbers suggested above constitute what might be considered a minimum number of objects that could in principle account for the general display as a whole. With an initial diameter in the size range of several meters, the putative parent object to the
Chant Meteor Procession might be taken as a fairly typical size for a TCO [18, 19], with such objects being encountered by the Earth on a decadal basis. What made the Chant Meteor Procession so spectacular and yet rare is that the parent object must have first undergone disaggregation at a time well before the individual components encountered the Earth's atmosphere, and that the entry angle for the meteoroid trajectories was very close to being horizontal. It is this low entry angle condition that dramatically reduces the probability of a long Chant Procession-like display coming about. Shoemaker [29] has shown that for an isotropic flux the probability $d P$ that the angle of entry $\theta$ will fall in the range $\Psi$ to $\Psi+d \Psi$ is independent of the gravity of the target body, and that $d P(\Psi<\theta<\Psi+d \Psi)=\sin (2 \Psi) d \Psi$, indicating that the most likely atmosphere entry angle is $45^{\circ}$. For the simulations presented here, in which the window for temporary Earth-orbit capture requires, at $100 \mathrm{~km}$ altitude, an entry angle between $6<\theta$ (deg.) $<7$ to the horizon, the probability of encounter is of order $\sim 0.5 \%$ of events.

Only a very few meteor processions have been witnessed over the past several centuries. That of August $18^{\text {th }}, 1783$ was observed to travel down the eastern coast of England and southward into European skies [30]. That of July 20 $0^{\text {th }}, 1860$ was witnessed from the Great Lakes region of the United States all the way to the Eastern seaboard [31]. The eyewitness accounts pertaining to these additional events present many similarities to those recorded for the Chant Procession, and the observations are suggestive of the possibility that extended procession-like events might be witnessed once every 70 to 100 years. To make further progress in understanding the origin and atmospheric interaction of procession-forming events, and to further determine their possible relationship to TCOs, a $21^{\text {st }}$ century display is now required with well-calibrated instrumental data being obtained with respect to energy, speeds, magnitudes and atmospheric trajectories [see e.g., 32, 33]. A typical nearEarth asteroid having a diameter of some 4 to 6 meters will deposit of order several tens of kilotons of equivalent TNT energy into the Earth's atmosphere during an encounter [34], and the total energy associated with the Chant or any other procession could hardly be much less than this. Such energies indicate that not only will optical, radio and radar observations likely be obtained for a procession-like event in the present day, but so too will infrasound data [35]. In addition to advanced ground-based instrumentation being available in the modern era, the ability of space-based detectors to track fragments as they move through the atmosphere [32] will additionally enhance the likelihood of finding meteoritic material on the ground [36]. Likewise, the coupling of meteoroid generated shock waves to the ground could potentially make for seismic, sonic boom, electrophonic sound, VLF radio and electronic interference data becoming available for analysis [13, 37, 38, 39]. On the historical basis that distinctive procession events occur at intervals of order 100 years, it is to be hoped that the next meteor procession will take place in the near future. 


\section{References}

[1] C. Chant, 1913. An extraordinary meteoric display. Journal of the Royal Astronomical Society of Canada, 7, 144-215.

[2] C. Chant, 1913. Further information regarding the meteoric display of February 9, 1913. Journal of the Royal Astronomical Society of Canada, 7, 438-447.

[3] W. F. Denning, 1913. Notes on the great meteoric stream of 1913, February $9^{\text {th }}$. Journal of the Royal Astronomical Society of Canada, 7, 404-413.

[4] W. H. Pickering, 1922. The meteoric procession of February 9, 1913. Popular Astronomy, 30, 632-637.

[5] W. H. Pickering, 1922. The meteoric procession of February 9, 1913- Part II. Popular Astronomy, 31, 96-104.

[6] A. D. Mebane, 1956. Observations of the great fireball procession of 1913 February 9, made in the United States. Meteoritics, 1, 405-421.

[7] D. W. Olson, and S. Hutcheon. 2013. The Great Meteor Procession of 1913. Sky and Telescope Magazine, February.

[8] J. G. Hills, and M. P. Goda, 1997. Meteoroids captured into Earth orbits by grazing atmospheric impacts. Planetary and Space Science, 45, 595-602.

[9] M. Beech. 2003. The Millman Fireball Archive I. Journal of the Royal Astronomical Society of Canada, 97, 71-77.

[10] M. Beech. 2004. The Millman Fireball Archive II. Sound reports. Journal of the Royal Astronomical Society of Canada, $99,34-41$.

[11] M. Beech, P. Brown, R. Hawkes., Z. Ceplecha., K. Mossman \& G. Wetherill. 1995. The fall of the Peekskill meteorite: Video observations, atmospheric path, fragmentation record and orbit. Earth, Moon and Planets, 68, 189-197.

[12] J. Borovicka, P. Spurny., P. Brown., P. Weigert, P. Kalenda., D. Clark \& L. Shrbeny. 2013. The trajectory, structure and origin of the Chelyabinsk asteroid impactor. Nature, 503, 235-237.

[13] M. Beech. 2014. Electrophonic Sound Generation from the Chelyabinsk Fireball. Earth, Moon and Planets, 113, 31-43.

[14] C. C. Wylie, 1939. The radiant and orbit of the meteors of February 9, 1913. Popular Astronomy, 6, 291-302.

[15] J. A. O'Keefe, 1959. A probable natural satellite: the meteor procession of February 9, 1913. Journal of the Royal Astronomical Society of Canada, 53, 59-65

[16] J. A. O'Keefe, 1964. Tektites and the Moon. NASA Technical Report:

https://ntrs.nasa.gov/archive/nasa/casi.ntrs.nasa.gov/19640005 370.pdf

[17] R. M. L. Baker, 1958. Ephemeral Natural Satellites of the Earth. Science, 128, 1211-1213.

[18] M. Granvik, J. Vaubaillon and R. Jedicke, 2012. The population of natural Earth satellites. Icarus, 218, 262-277.

[19] G. Fedorets, M. Granvik and R. Jedicke, 2017. Orbit and size distributions for asteroids temporarily captured by the EarthMoon system. Icarus, 285, 83-94.
[20] JPL Small-body database browser: https://ssd.jpl.nasa.gov/sbdb.cgi?sstr=2006RH120.

[21] D. L. Clark et al., 2016. Impact detection of temporarily captured natural satellites. The Astronomical Journal, 151: 135 (15pp).

[22] M. Beech. 2014. Grazing impacts upon Earth's surface: towards an understanding of the Rio Cuarto crater field. Earth, Moon and Planets, 113, 43-71.

[23] R. Clift, J. R. Grace, and M. E. Weber, 2005. In Bubbles, Drops and Particles, Academic Press, New York.

[24] H. J. Melosh and T. J. Goldin, 2008. Lunar and Planetary Science XXXIX, 2457.pdf

[25] J. M. Picone, A. E. Hedin, D. P. Drob, A. C. Aiken, 2002. NRLMSISE-00 empirical model of the atmosphere: statistical comparisons and scientific issues. Journal of Geophysical Research (Space Physics), 107, SIA15.

[26] O. Popova, et al., 2011. Very low strengths of interplanetary meteoroids and small asteroids. Meteoritics and Planetary Science, 46, 1525-1550.

[27] C. S. L. Keay. 1992. Electrophonic sounds from large meteor fireballs. Meteoritics and Planetary Science, 27, 144-148.

[28] Z. Ceplecha, 1994. Earth-grazing daylight fireball of August 10, 1972. Astronomy and Astrophysics, 283, 287-288.

[29] E. Shoemaker, 1962. In, Physics and Astronomy of the Moon. Kopal, Z (Ed.). Academic Press, New York. p. 283.

[30] M. Beech, 1989. The Great Meteor of August $17^{\text {th }}, 1783$. Journal of the British Astronomical Association, 99, 130-134.

[31] D. W. Olson et al., 2010. Walt Whitman's Year of Meteors. Sky and Telescope, July.

[32] P. Chodas. https://cneos.jpl.nasa.gov/fireballs/intro.html (accessed May, 2018).

[33] W. Cooke. https://fireballs.ndc.nasa.gov/ (accessed May 2018).

[34] M. Zolensky, P. Bland, P. Brown, and I. Halliday. 2006. In Meteorites and the Early Solar System II. Edited by Dante S. Lauretta and Harold Y. McSween Jr. University of Arizona Press.

[35] N. Gi, and P. Brown. 2017. Refinements of bolide characteristics from infrasound measurements. https://arxiv.org/ftp/arxiv/papers/1704/1704.07794.pdf

[36] P. Bland. 2015. Catching a falling star (or meteorite) - Fireball camera networks in the $21^{\text {st }}$ century. Elements magazine, 160161, June.

[37] C. Caudron, et al., 2016. Infrasound and seismic detections associated with the 7 September 2015 Bangkok fireball. Geoscience letters, 3:26.

[38] O. Popova, et al., 2013. Chelyabinsk Airburst, Damage Assessment, Meteorite Recovery, and Characterization. Science. 342, 1069-1073.

[39] W. Edwards, D. Eaton, and P. Brown. 2008. Seismic observations of meteors: coupling theory and observations. Reviews of Geophysics, 46, RG4007. 